\title{
Shot noise and transport in small quantum cavities with large openings
}

\author{
R. G. Nazmitdinov, ${ }^{1,2,3}$ H.-S. Sim, ${ }^{1, *}$ H. Schomerus, ${ }^{1}$ and I. Rotter ${ }^{1}$ \\ ${ }^{1}$ Max-Planck-Institut für Physik komplexer Systeme, D-01187 Dresden, Germany \\ ${ }^{2}$ Departament de Física, Universitat de les Illes Balears, E-07071 Palma de Mallorca, Spain \\ ${ }^{3}$ Bogoliubov Laboratory of Theoretical Physics, Joint Institute for Nuclear Research, 141980 Dubna, Russia
}

(Received 29 August 2002; published 17 December 2002)

\begin{abstract}
We present a dynamical analysis of the transport through small quantum cavities with large openings. The systematic suppression of shot noise is used to distinguish direct, deterministic from indirect, indeterministic transport processes. The analysis is based on quantum mechanical calculations of $S$ matrices and their poles for quantum billiards with convex boundaries of different shape and two open channels in each of the two attached leads. Direct processes are supported when special states couple strongly to the leads, and can result in deterministic transport as signified by a striking system-specific suppression of shot noise.
\end{abstract}

DOI: 10.1103/PhysRevB.66.241302

PACS number(s): 73.23.Ad, 05.45.Mt, 72.70.+m

Transport through quantum devices is one of the topical subjects in mesoscopic physics. The diagrammatic perturbation theory and the random-matrix theory (RMT) predict that the conductance varies from sample to sample by a universal small amount, if details of the quantum device and its coupling to the electronic reservoirs are not important ${ }^{1,2}$ (for reviews see Refs. 3 and 4). These universal conductance fluctuations have been observed experimentally for disordered quantum wires ${ }^{5}$ and quantum dots. ${ }^{6}$ The RMT assumes that the dwell time $\tau_{\text {dwell }}$ of electrons in the system is large enough to wash out all system-specific details, and supposes that the conductance is determined by a random coupling of the scattering states to the leads. Recent studies clearly demonstrate that the conductance of small quantum dots with very large openings, which violate the RMT conditions, indeed only can be fully understood on the basis of their specific properties. ${ }^{7,8}$ This is the deep quantum mechanical regime, in which the Heisenberg time $\tau_{\mathrm{H}}=\hbar / \Delta$ becomes a relevant time scale for the internal dynamics, where $\Delta$ is the mean resonance spacing. Under these conditions the coupling between specific states of the cavity and the channel wave functions depends sensitively and nonuniversally on the position of the attached leads. ${ }^{9,10}$ For instance, an appropriate attachment of the leads to the Bunimovich stadium billiard, a paradigm for quantum chaotic systems, gives rise to a family of short-lived whispering gallery modes (WGM) related to the shortest classical paths connecting both leads. These special states have small lifetimes $\tau_{\text {dwell }} \ll \tau_{\mathrm{H}}$ and correspond to broad overlapping resonances, which influence the transport over a large range of energies. As a consequence, the conductance of the quantum cavity significantly exceeds the prediction of RMT. ${ }^{10}$

It is well established that shot noise (the zero-frequency current-current correlations caused by the discreteness of electric charge) provides valuable complementary information not contained in the conductance (for a review see Ref. 11). It was found that correlations due to Fermi statistics suppress the shot-noise power $P$ by a factor $\mathcal{F}=P / P_{0}$ below the maximal value $P_{0}=2 e G_{0} V$ of incoherent transport, with $e$ the unit of charge, $G_{0}$ the series conductance of the two point contacts, and $V$ the applied voltage. The universal predictions for the shot-noise suppression factor are $\mathcal{F}=1 / 3$ for noninteracting electrons in diffusive wires ${ }^{12,13}$ and $\mathcal{F}=1 / 4$ for quantum dots, ${ }^{14,15}$ respectively.

Classically, the zero-temperature shot noise vanishes because of the deterministic nature of classical transport. Based on the semiclassical approach to quantum transport in classically chaotic systems, it was predicted that the shot noise indeed can be reduced below $\mathcal{F}=1 / 4$ if the Ehrenfest time $\tau_{E}$ (the time scale for wave packets to dissolve due to chaotic dynamics) is larger than $\tau_{\text {dwell }}{ }^{16}$ Recently, this prediction has been verified experimentally by using chaotic cavities, where the time that electrons dwell inside can be tuned. ${ }^{17}$ The analog suppression in soft chaotic systems has been considered in Ref. 18.

In the present paper we investigate the shot noise in the deep quantum mechanical regime, which is opposite to the semiclassical limit of Refs. 16-18. We find that the bands of broad resonances (formed by appropriate attachment of the leads) support direct, deterministic transport channels with dwell times $\tau_{\text {dwell }}$ less than the time $\tau_{\text {indet }}$ that separates deterministic from indeterministic dynamics (in the quantum regime, this time scale depends on the detailed dynamical exploration of the system, and only may be identified with the Ehrenfest time in the long time, semiclassical limit). This is signified by a striking suppression of the shot noise, even when the corresponding classical dynamics in the quantum cavity is chaotic. On the other hand, resonances with large dwell times are indeterministic and contribute to the shot noise as predicted by RMT. The general observation of shotnoise suppression by direct processes is consistent with the work of Baranger and Mello, ${ }^{19}$ who found that in their presence the transmission eigenvalues are redistributed towards the deterministic values 0,1 . We provide a detailed analysis of the dynamical aspects involved in the formation of the corresponding deterministic processes, by means of the resulting shot-noise suppression and of a direct investigation of the resonance wave functions.

In order to study the role of special states for the transport in small quantum systems with large openings, we consider cavities with convex boundaries and two attached leads, where each lead supports $N=2$ propagating modes. We analyze two stadium billiards of Bunimovich type [length of the linear segment $L=3 \pi /(\pi+1)$ and radius $R=L$ ] with differ- 

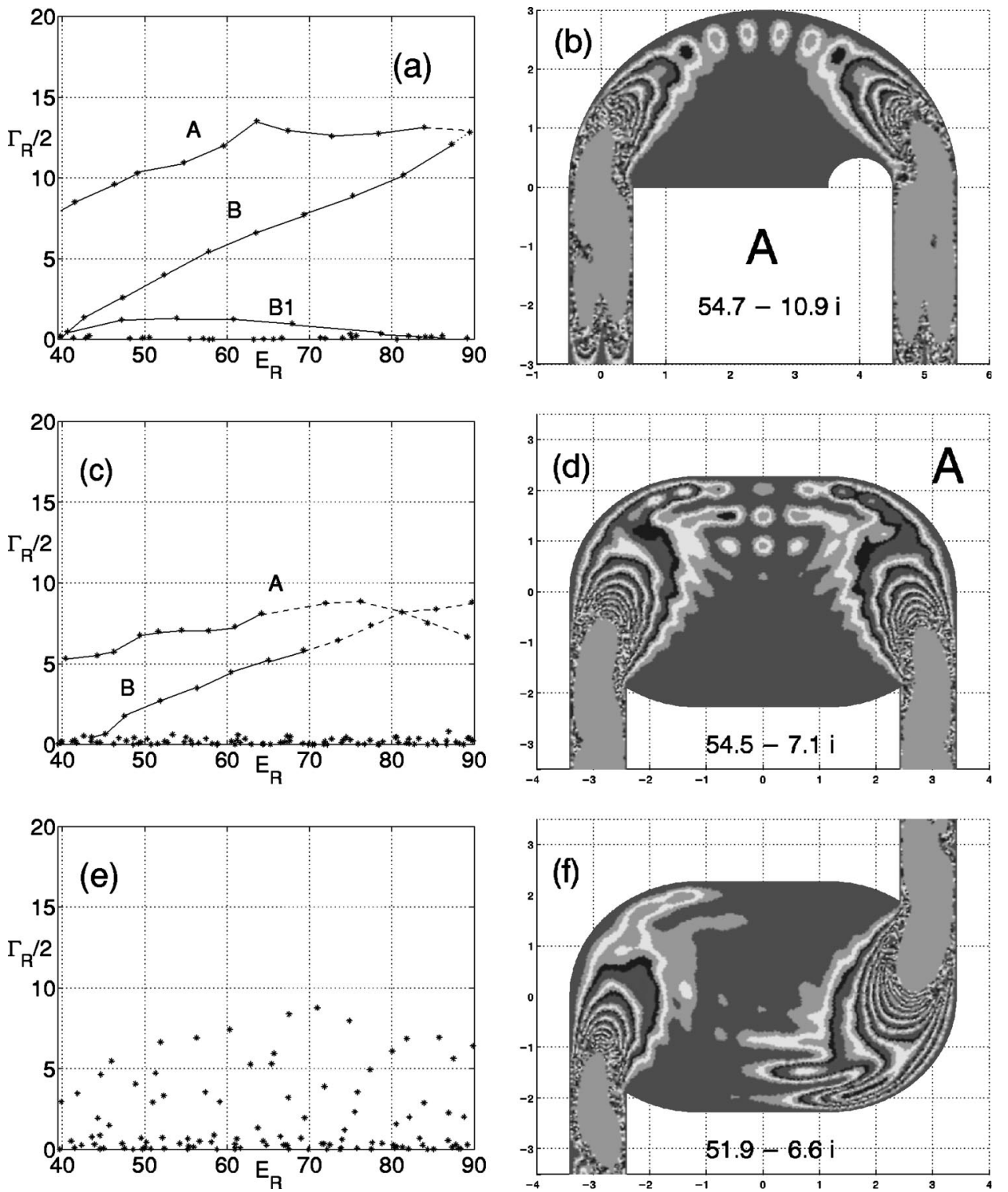

FIG. 1. The poles $\mathcal{E}_{R}=E_{R}$ $-i \Gamma_{R} / 2$ of the $S$ matrix (left panels) and representative pictures $\left|\Phi_{R}\right|^{2}$ of the wave functions of short-lived states (right panels) for the billiards SIS (a, b), Bun1 (c, d), and Bun2 (e, f). Poles of the $S$ matrix (denoted by stars) far from the real axis are connected by lines for guiding the eye. The resonance states are shown for the energy interval $4 \pi^{2} \leqslant E \leqslant 9 \pi^{2}$, between the second and the third threshold for propagating modes in the leads. The energies and widths are in units $\hbar^{2} /\left(2 m d^{2}\right)$ $=1$, where $d=1$ is the width of the leads. ent positions of the leads [Bun1 and Bun2, see Fig. 1(d) and $1(\mathrm{f})]$, and also consider a semicircle $(R=3)$ with an internal scatterer [SIS, see Fig. 1(b)]. The Bunimovich stadium is a prototype of chaotic motion, while the SIS is most suitable for the propagation of the WGM. ${ }^{9}$

The poles of the $S$ matrix are obtained by the method of exterior complex scaling ${ }^{20}$ in combination with the finite element method (for details see Ref. 21). The $S$ matrix itself is calculated in small energy steps by directly solving the Schrödinger equation in a discretized space, according to the method suggested in Ref. 22. The poles of the $S$ matrix obtained by the numerical calculation are shown in the left panels of Fig. 1. The plots demonstrate the formation of bands of overlapping resonances denoted by $A$ and $B$ for the SIS and the Bun1 billiard, while no evident band is formed in the Bun 2 billiard. Another band $B 1$ of resonances can be identified for the SIS, but these resonances do not overlap.

The bands of overlapping resonances in the SIS and in the Bun1 billiard are the result of resonance trapping, ${ }^{23}$ a phenomenon in which few states accumulate the major part of the total sum of the widths $\sum_{R} \Gamma_{R}(E)$ (which is fixed by a sum rule; for quantum billiards see Refs. 9 and 10). The states from band $A$ and $B$ are related to the first and second transverse excitation of the propagating modes in the leads, respectively. The resonance wave function $\left|\Phi_{R}(x, y)\right|^{2}$ displays a strong localization along the convex boundary, which is a characteristic feature of the WGM. ${ }^{10}$ In the SIS, these trajectories almost exclusively correspond to one bounce at the convex boundary, while for the Bun1 billiard, there is also a small contribution of trajectories with two bounces. In the SIS the WGM accumulate $R=\sum \Gamma_{W G M} / \Sigma_{i} \Gamma_{i}>98 \%$ of the total sum of widths $\Sigma \Gamma_{i}$ of all states, while in the Bun1 billiard they accumulate a fraction $R=93 \%$ of the total sum. For these direct processes one can suppose that $\tau_{\text {indet }}$ is larger than the dwell time $\tau_{\text {dwell }}$, and this will be demonstrated, indeed, by our dynamical analysis.

The bands of WGM are inhibited in the Bun2 billiard [Fig. 1(e) and 1(f)] by destructive interference, since the coupling matrix elements of the WGM with the channel wave functions have different phases for different leads. As we 


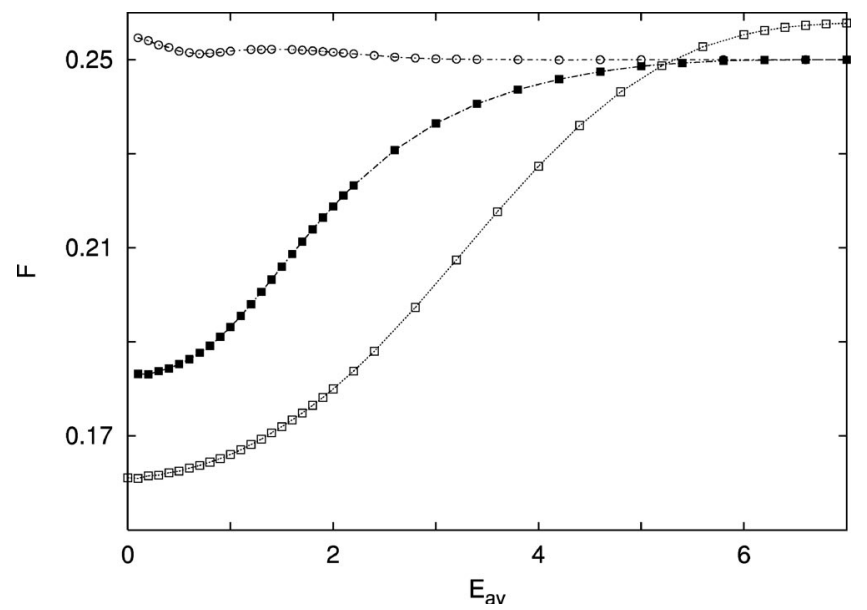

FIG. 2. Shot-noise suppression factor $\mathcal{F}$ vs the value of the energy-averaging interval $E_{\text {av }}$ for the three different billiards SIS (open square), Bun1 (filled square), and Bun2 (open circle).

will demonstrate, the resonances in the Bun2 comply with $\tau_{\text {indet }}<\tau_{\text {dwell }}$, corresponding to indeterministic processes. This is also the case for the long-living resonances in the SIS and Bun1 billiards.

The suppression factor $\mathcal{F}=(2 / N) \operatorname{tr}[T(1-T)]$ is determined by the eigenvalues (transmission probabilities) of the matrix $T=t^{\dagger} t$, where $t$ is the transmission matrix. ${ }^{13,24}$ The closer the transmission probabilities are to the classical values 0 or 1 of completely deterministic dynamics, the smaller is the shot noise. To get a thorough insight into the interplay of deterministic and indeterministic dynamics, we employ the procedure recently developed in Ref. 18. The major element of this procedure is the purposeful replacement of system-specific dynamics with dwell times $\tau_{\text {dwell }} \gtrsim \tau_{\text {av }}$ by indeterministic processes. In the first step, the system-specific details are eliminated by averaging the scattering matrix $S$ over an energy range $\left[E_{0}-E_{\mathrm{av}} / 2, E_{0}+E_{\mathrm{av}} / 2\right]$ of width $E_{\mathrm{av}}$ $=\hbar / \tau_{\mathrm{av}}$,

$$
\bar{S}\left(E_{\mathrm{av}} ; E_{0}\right)=\frac{1}{E_{\mathrm{av}}} \int_{E_{0}-E_{\mathrm{av}} / 2}^{E_{0}+E_{\mathrm{av}} / 2} S(E) d E .
$$

Indeterministic processes then are introduced by coupling the system to an auxiliary indeterministic system, with a scattering matrix $S_{0}$ taken from the appropriate circular ensemble of RMT. The composed system with scattering matrix

$$
S^{\prime}\left(E_{\mathrm{av}} ; E_{0} ; S_{0}\right)=\bar{S}\left(E_{\mathrm{av}} ; E_{0}\right)+\mathcal{T}^{\prime}\left(1-S_{0} \mathcal{R}\right)^{-1} S_{0} \mathcal{T}
$$

is a member of the Poisson kernel of RMT. ${ }^{19,25,26}$ The matrix $S^{\prime}$ must be unitary, which determines the coupling matrices $\mathcal{T}, \mathcal{T}^{\prime}$, and $\mathcal{R}$. We numerically calculate the mean suppression factor $\mathcal{F}\left(E_{\text {av }}\right)$ for fixed values of $E_{\text {av }}$ by first averaging over the random matrix $S_{0}$ within each Poisson kernel, and then averaging over $E_{0}$ within a range $\left(E_{0}^{\min }, E_{0}^{\max }\right)$.

The result for the suppression factor $\mathcal{F}\left(E_{\mathrm{av}}\right)$ in the three billiards is shown in Fig. 2. Here $E_{0}^{\min }=40$ and $E_{0}^{\max }=90$ are fixed to the values where the second and the third propagating mode opens in each lead, respectively. The value $\mathcal{F}\left(E_{\text {av }}\right.$ $=0)$ is the shot-noise suppression factor of the billiards

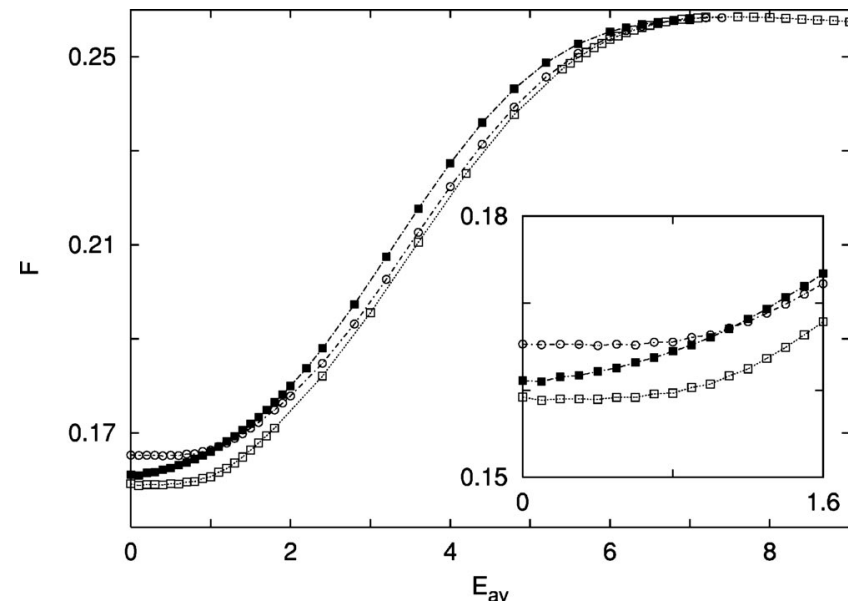

FIG. 3. Shot-noise suppression factor $\mathcal{F}$ of the SIS billiard as a function of the energy-averaging interval $E_{\mathrm{av}}$. Different curves correspond to the different lower-energy bounds $E_{0}^{\mathrm{min}}=40$ (filled square), $E_{0}^{\mathrm{min}}=52.5$ (open circle), and $E_{0}^{\min }=65$ (open square).

themselves, without any modification of their dynamics. The shot noise in the billiard Bun2 corresponds well to the universal prediction $\mathcal{F}=1 / 4$ of RMT, from which we conclude that $\tau_{\text {dwell }} \gtrsim \tau_{\text {indet }}$ and that the dynamics is indeterministic. For the other two billiards, the suppression factor is significantly reduced below the universal value. We now argue that this can be attributed to the WGM, which support deterministic transport.

The modes that lead to shot-noise reduction can be identified from the dependence of $\mathcal{F}$ on $E_{\text {av }}$, which indicates the sensitivity to replacement of system-specific processes by indeterministic ones. For the billiards SIS and Bun1, the function $\mathcal{F}$ increases monotonically with increasing contribution of the random dynamics (increasing $E_{\text {av }}$ ) and eventually approaches the universal prediction of RMT. However, the slope of $\mathcal{F}\left(E_{\text {av }}\right)$ for small $E_{\text {av }}$ almost vanishes. Hence, without any consequences for the shot noise the long-living resonances (trapped by the WGM) can be replaced by (and hence are equivalent to) indeterministic processes. On the other hand, the suppression factor rises significantly when the energy-averaging window $E_{\text {av }}$ becomes of the order of the width $\Gamma_{R}$ of the WGM modes in each of the two billiards (cf. Fig. 1), which hence support deterministic transport.

The position of the attachment of the leads plays a decisive role, since it determines the character of the systemspecific broad resonances. In the Bun2 billiard, the suppression factor $\mathcal{F}$ is almost independent of $E_{\mathrm{av}}$ and hence completely insensitive to the replacement of system-specific properties by indeterministic dynamics. Even the short-living resonances no longer correspond to deterministic classical transport. The remarkable difference in the behavior of the suppression factor $\mathcal{F}$ for the Bun 1 and Bun 2 billiards arises from the different position of the leads attached to the same quantum system, which results in the formation of direct, deterministic transport channels in the first case, while no such channels are formed in the second case.

Both in the SIS and in the Bun1 billiard, two bands $A, B$ of broad resonances have been identified. We now analyze 
these bands in more detail for the SIS. Figure 3 displays the result for the suppression factor for three different values of $E_{0}^{\min }$, while $E_{0}^{\max }=90$ remains fixed to the value where the third propagating mode opens in the leads. In this way we focus on the contribution of the WGM from different parts of the bands. The lowest and the highest suppression factor $\mathcal{F}$ are obtained if we only consider WGM with energies $E_{R}$ $\geqslant E_{0}^{\mathrm{min}}=65$ and $E_{R} \geqslant E_{0}^{\mathrm{min}}=53$, respectively [see Fig. 1(a) and the inset in Fig. 3]. The difference between the largest and the smallest values is small, since the dynamics is mainly determined by the states of the band $A$, which are strongly localized along the convex boundary and all have similar width. The widths of the states from the family $B$ depend almost linearly on their energy, and become small when one approaches the threshold $E_{R}=40$ of the second propagating mode in the leads. For $E_{0}^{\mathrm{min}}=40$ the resonances

*Present address: Korea Institute for Advanced Study, 207-43

Cheongryangri-dong, Dongdaemun-gu, Seoul 130-012, Korea.

${ }^{1}$ B.L. Altshuler, Pis'ma Zh. Éksp. Teor. Fiz. 41, 530 (1985) [JETP Lett. 41, 648 (1985)]; P.A. Lee and A.D. Stone, Phys. Rev. Lett. 55, 1622 (1985).

${ }^{2}$ R. Blümel and U. Smilansky, Phys. Rev. Lett. 60, 477 (1988); R.A. Jalabert, H.U. Baranger, and A.D. Stone, Phys. Rev. Lett. 65, 2442 (1990).

${ }^{3}$ C.W.J. Beenakker, Rev. Mod. Phys. 69, 731 (1997).

${ }^{4}$ Y. Alhassid, Rev. Mod. Phys. 72, 895 (2000).

${ }^{5}$ S. Washburn and R.A. Webb, Adv. Phys. 35, 375 (1986); R.A. Webb and S. Washburn, Phys. Today 41(12), 46 (1988).

${ }^{6}$ I.H. Chan, R.M. Clarke, C.M. Marcus, K. Campman, and A.C. Gossard, Phys. Rev. Lett. 74, 3876 (1995).

${ }^{7}$ R. Akis, D.K. Ferry, and J.P. Bird, Phys. Rev. B 54, 17705 (1996); J.P. Bird, R. Akis, D.K. Ferry, D. Vasileska, J. Cooper, Y. Aoyagi, and T. Sugano, Phys. Rev. Lett. 82, 4691 (1999).

${ }^{8}$ I.V. Zozoulenko, R. Schuster, K.-F. Berggren, and K. Ensslin, Phys. Rev. B 55, 10209 (1997); T. Blomquist and I.V. Zozoulenko, ibid. 61, 1724 (2000).

${ }^{9}$ R.G. Nazmitdinov, K.N. Pichugin, I. Rotter, and P. Šeba, Phys. Rev. E 64, 056214 (2001).

${ }^{10}$ R.G. Nazmitdinov, K.N. Pichugin, I. Rotter, and P. Šeba, Phys. Rev. B 66, 085322 (2002).

${ }^{11}$ Ya.M. Blanter and M. Büttiker, Phys. Rep. 336, 1 (2000). of type $B$ with smallest width are included into the averaging interval, and the slope of the suppression factor $\mathcal{F}\left(E_{\mathrm{av}}\right)$ for very small values of $E_{\text {av }}$ increases noticeably. This shows that even the longest-living resonances in family $B$ support system-specific direct, deterministic processes.

Summarizing, we analyzed the transport through small quantum cavities with large openings, deep in the quantum regime. We observed an essential suppression of the shot noise when the transport is dominated by system-specific broad resonances that support direct processes well described by deterministic classical dynamics. These deterministic transport channels can exist even if the closed system manifests typical properties of chaotic dynamics, and their formation sensitively depends on the precise position of the leads.

We gratefully acknowledge discussions with Pier A. Mello, as well as with Konstantin Pichugin.

${ }^{12}$ K.E. Nagaev, Phys. Lett. A 169, 103 (1992).

${ }^{13}$ C.W.J. Beenakker and M. Buttiker, Phys. Rev. B 46, 1889 (1992).

${ }^{14}$ H.U. Baranger and P.A. Mello, Phys. Rev. Lett. 73, 142 (1994).

${ }^{15}$ R.A. Jalabert, J.-L. Pichard, and C.W.J. Beenakker, Europhys. Lett. 27, 255 (1994).

${ }^{16}$ O. Agam, I. Aleiner, and A. Larkin, Phys. Rev. Lett. 85, 3153 (2000).

${ }^{17}$ S. Oberholzer, E.V. Sukhorukov, and C. Schönenberger, Nature (London) 415, 765 (2002).

${ }^{18}$ H.-S. Sim and H. Schomerus, Phys. Rev. Lett. 89, 066801 (2002).

${ }^{19}$ H.U. Baranger and P.A. Mello, Europhys. Lett. 33, 465 (1996); P.A. Mello and H.U. Baranger, Waves Random Media 9, 105 (1999).

${ }^{20}$ N. Moiseyev, Phys. Rep. 302, 211 (1998).

${ }^{21}$ E. Persson, K. Pichugin, I. Rotter, and P. Šeba, Phys. Rev. E 58, 8001 (1998); P. Šeba, I. Rotter, M. Müller, E. Persson, and K. Pichugin, ibid. 61, 66 (2000); I. Rotter, E. Persson, K. Pichugin, and P. Šeba, ibid. 62, 450 (2000).

${ }^{22}$ T. Ando, Phys. Rev. B 44, 8017 (1991).

${ }^{23}$ I. Rotter, Rep. Prog. Phys. 54, 635 (1991).

${ }^{24}$ G.B. Lesovik, Pis'ma Zh. Eksp. Teor. Fiz. 49, 513 (1989) [JETP Lett. 49, 592 (1989)].

${ }^{25}$ P.A. Mello, P. Pereyra, and T.H. Seligman, Ann. Phys. (N.Y.) 161, 254 (1985).

${ }^{26}$ P.W. Brouwer, Phys. Rev. B 51, 16878 (1995). 